\title{
Dialysis after graft loss: a Swiss experience
}

Marco Bonani ${ }^{1^{*}}$, Rita Achermann ${ }^{2}$, Harald Seeger ${ }^{1}$, Michael Scharfe ${ }^{3}$, Thomas Müller ${ }^{1}$, Stefan Schaub ${ }^{3}$, Isabelle Binet ${ }^{4}$, Uyen Huynh-Do ${ }^{5}$, Suzan Dahdal ${ }^{5}$, Dela Golshayan ${ }^{6}$, Karine Hadaya ${ }^{7}$, Rudolf Wüthrich ${ }^{1}$, Thomas Fehr ${ }^{8}$, Stephan Segerer ${ }^{1,9}$, and the Swiss Transplantation Cohort Study (STCS)

${ }^{1}$ Division of Nephrology, University Hospital Zürich, Switzerland

2Department Transplant Immunology and Nephrology, University Basel Hospital, Switzerland

${ }^{3}$ Clinical Trial Unit, Department of Clinical Research, University Basel Hospital, Switzerland

${ }^{4}$ Division of Nephrology/Transplantation Medicine, Kantonsspital St. Gallen, Switzerland

${ }^{5}$ Department of Nephrology and Hypertension, University of Bern, Switzerland

${ }^{6}$ Transplantation Center, CHUV University Hospital, Lausanne, Switzerland

${ }^{7}$ Division of Nephrology, Geneva University Hospitals, Geneva, Switzerland

${ }^{8}$ Department of Internal Medicine, Kantonsspital Graubünden, Chur, Switzerland

${ }^{9}$ Division of Nephrology, Dialysis and Transplantation, Kantonsspital Aarau, Aarau Switzerland

Corresponding Author:

Stephan Segerer, MD

eMBA UZH

Leitender Arzt Nephrologie

Kantonsspital

Tellstrasse 25

$\mathrm{CH}-5001$ Aarau

Tel: $\quad++41628389574$

Fax: $\quad++41628386552$

Short title: Dialysis after graft loss

Keywords: Kidney transplantation, graft loss, dialysis, immunosuppression, allograft nephrectomy

Word count: 
Abstract: $x x$

Text: $x x$ 


\section{Abstract}

Renal transplantation is the treatment of choice for suitable patients with end-stage renal disease. Patients who return to dialysis after graft loss (DAGL), have a high early morbidity and mortality. As there are major differences in the treatment protocols of patients returning to dialysis, we used data from the Swiss Transplant Cohort Study (STCS) to describe current management and outcome in Switzerland.

We included 1499 patients who received a renal allograft between May 2008 and end of December 2014. 78 patients lost the graft during follow up, of those 41 allografts were lost within one year after transplantation (53\%). Eleven patients died within 30 days after allograft loss. Patient survival was $86 \%, 81 \%$ and $74 \%$ after 30,90 and 365 days after graft loss respectively. After graft loss, $90 \%$ of the patients started hemodialysis (31\% with catheters, $54 \%$ with native fistulae, and $10 \%$ with vascular grafts). Starting with a permanent vascular access was associated with a decreased mortality (hazard ratio 0.32 ). At the time of graft loss, the majority of patients were on triple immunosuppressive therapy, which was reduced to double immunosuppression during the following year. After allograft nephrectomy, immunosuppression was significantly reduced. One year after graft loss 31 patients in the early and 16 in the late loss group had been nephrectomized. Three years after graft loss, $37 \%$ of the patients with early and $9 \%$ with late graft loss received another allograft, of those 20 out of 23 had an allograft nephrectomy.

In summary, we present a detailed analysis of the STCS of patients after allograft failure. Our data illustrate a high mortality, a high number of allograft nephrectomies and retransplantations. Patients commencing hemodialysis with a catheter had a significantly higher mortality compared to patients with a definitive vascular access. The role of 
immunosuppression reduction and allograft nephrectomy as interdependent factors for mortality and re-transplantation needs further evaluation. 


\section{Introduction}

Dialysis and renal transplantation are currently integrated into a long-term treatment plan for patients with renal failure. A comprehensive pre-dialysis evaluation reduces the mortality of incident dialysis patients and increases the chance of receiving an allograft [1, 2]. Data suggests that the pre-dialysis treatment might be suboptimal for patients with dialysis after graft loss (DAGL) as compared to incident dialysis patients [3].

In the first year after transplantation about $7 \%$ cadaveric donor recipients and $3 \%$ of the living donor recipients return to dialysis (USRDS, 2016). The period after the loss of a renal allograft is very important for the patient, as mortality and morbidity in the first months, as well as in the long run are particularly high [1, 2]. The death rate of US allograft recipients was found to be $6.4 / 100$ patient-years on the waiting list, and in the peritransplant period it was higher with 8.2/100 patient-years [4]. Initiation of dialysis after allograft loss was associated with a strikingly high death rate of $17.9 / 100$ patient-years, more than twice the number of the period after major surgery [4]. Little information is available from European transplant centers about the current treatment of patients returning to dialysis.

Here we have used the data from the Swiss Transplantation Cohort Study (STCS) to define patients who lost the allograft and collected data about dialysis, immunosuppressive treatment, allograft nephrectomy and death after the loss of the allograft.

\section{Materials and Methods}

\section{Study Population}

For the present study, we analyzed patients enrolled in the STCS, a nationwide, longitudinal multicenter study. All patients who received a renal allograft in Switzerland between Mai between 2008 and December 2014 were included. All patients were at least 18 years of age, 
and had given informed consent. Patients with primary non-function or additional organs transplanted were excluded. Overall 1499 patients received a renal allograft (first allograft or re-transplantation) of whom 78 lost their allograft at least once until January 2015. As we considered only the first graft-loss of a patient within STCS, 2 graft losses were excluded. Data analyzed for the present study are part of the STCS data collection for the period up to graft loss. Thereafter, no further data was collected by the STCS, thus information on type of dialysis, the vascular access, immunosuppressive therapy, allograft nephrectomy and available information on cause of death after graft loss were collected in addition by each allograft center for patients with graft loss. Data were collected up to the time point of death or re-transplantation of the patient or time point of data collection (July 2015 until January 2016). The data collection was approved by the Zurich ethical committee for research involving human subjects (KEK-ZH-Nr. 2015-0445).

\section{Statistical analysis}

For the analysis, the patients with graft loss were divided into two groups depending on whether the graft loss occurred within one year after transplantation or later. To describe the course after graft loss, a multistate model with initial state dialysis after graft loss, transient state nephrectomy and absorbing states death or re-transplantation was set up (R-library mstate). Confidence intervals for the probabilities to be in a particular state at a selected time point were derived with the bootstrap method.

To compare mortality between patients with and without graft loss, a cox proportional hazard model with a time dependent covariate for graft loss was set up. As the risk of death depends on the time after graft loss, a categorical time dependent covariate was incorporated with categories no graft loss, $0-30,31-90,91-180$, more than 180 days after graft loss. To test 
whether the model adequately represents the data, a test for violation of cox proportional hazard assumptions was performed and Schoenfeld residuals plots were inspected.

All analyses were performed using R Statistical Software Version 3.3.1 (Core Team. R: A Language and Environment for Statistical Computing. Vienna, Austria; 2016. Available online: https://www.r-project.org/).

\section{Results}

\section{Patient characteristics}

Characteristics of the study population, separated into patients with and without graft loss, were illustrated in Figure 1 and Table 1. A total of 78 patients lost at least one renal allograft. Patients with graft loss were further divided into an early graft loss group (41 patients, 53\%) with a graft loss within 365 days after transplantation and a late group with a graft loss later than 365 days after transplantation (37 patients, 47\%). The median follow up time for patients without graft loss was 1445 (Inter Quartile Range (IQR) 729, 1908) days and for patients with graft loss 1186 (IQR 463, 2244) days up to time point of graft loss (Table 2). There were no significant differences in the baseline characteristics according to age, type of dialysis, reason for transplantation and comorbidities between patients with and without graft loss at the time of transplantation. In the group with allograft loss, there were significantly less patients who received an allograft from living donors and less patients who received a preemptive allograft $(p<0.001)$.

\section{Mortality of patients after graft loss}

After graft loss, patients were followed for a median of 446 days in the early group (IQR 137, $925)$ and for 790 days for the late group (IQR 441, 1468). For each patient and point of 
time, one of the following statuses were assigned: on dialysis without nephrectomy, on dialysis with nephrectomy, death or re-transplanted. The result of this multistate model is shown in Figure 2 and in Table 2. The percentage of patients in each state is illustrated in Figure 3.

During the observation period $23(29 \%)$ patients died: 13 patients who lost the allograft early and 10 patients in the group with allograft loss after 1 year (Figure 2). In the early group 6 out of 41 patients died within 30 days, in the late group 5 out of 37 . Six patients died after a nephrectomy with four in the early and two in the late group. The transition probability for dying after nephrectomy was $12 \%$.

A Kaplan Meier curve for death after graft loss (Figure 4) shows, that immediately after graft loss the risk of dying is high, survival rate was $86 \%$ [CI 95\% 76-92], 81\% [CI 95\% 70-88] and $74 \%$ [Cl 95\% 63-83] after 30, 90 days and 1 year after graft loss respectively.

A cox proportional hazard model adjusted for age and diabetes with a categorical time dependent variable for the time since graft loss showed a hazard ratio (HR) of $87.7(\mathrm{Cl} 95 \%$ 45-179) for the period 0-30 days after graft loss compared to patients with no graft loss (Figure 4). For the period 180 days after graft loss, HR decreased to 2.7 (Cl 95\% 1.2-6.2). No significant difference was observed between early and late graft loss group. Causes of death were cardiovascular disease $(n=8 / 23)$, infectious complications $(n=4 / 23)$, and 9/23 died of other reasons (not further specified). Mortality was associated with age (HR per 10 years 1.7, Cl 95\% 1.4-2.0, p value < 0.0001) and diabetes status (HR 2.5, Cl 95\% 1.7-3.7, $p$ value $<0.0001)$ at time of transplantation.

\section{Allograft nephrectomy}


A total of 50 nephrectomies were performed in the 78 patients, 31 in the early group and 19 in the late group. In the early group all surviving patients were nephrectomized with a single exception. 19 of $31(61 \%)$ nephrectomies were performed at time of graft loss in the early group, while only 3 of $19(16 \%)$ allografts were removed immediately in the late group. Half a year after graft loss, $76 \%(\mathrm{Cl} 95 \% 59-86)$ of patients in the early and $35 \%(\mathrm{Cl} 95 \% 20-51)$ in the late group were nephrectomized as shown by the cumulative incidence graph adjusted for competing risk re-transplantation and death (Figure 5). As some individuals died, only $5 \%$ patients of the early group were on dialysis without nephrectomy half a year after graft loss compared to $49 \%$ in the late group. Thereafter, no further nephrectomies were performed. At two years after graft loss, only $24 \%$ of patients were left without nephrectomy in the late group as illustrated by the multistate model (Figure 3). The reasons for nephrectomy are listed in Table 3. Infection and rejection were similar in number as causes given for nephrectomy.

\section{Retransplantation}

The earliest retransplantation was performed 327 days after graft loss. Retransplantation was associated with the time to graft loss. Patients with early graft loss were more likely to have a retransplantation within the first 3 years. Three years after graft loss, $25.4 \%$ of patients in the early group $(\mathrm{Cl} 95 \% 15.6$-37.4) were re-transplanted and $8.8 \%(\mathrm{Cl} 95 \% 0.0$ - 24.8) in the late group. Most of the retransplanted patients underwent a nephrectomy of the previous allograft (20 out of 23 ).

\section{Type of dialysis and permanent vascular access at the start of dialysis}


After the loss of graft function the vast majority of patients started on hemodialysis $(n=70)$, only three patients started on peritoneal dialysis, and for another three patients no information was available. Two patients decided not to start renal replacement therapy (2 of $78,2.6 \%)$, one from each the early and late group. A total of 38 of 70 patients $(54.3 \%)$ started dialysis with a native fistula, with $22 / 37$ in the early (59.5\%) and 16/33 (48.5\%) in the late group. Four patients in the early and two patients in the late group started with a functioning vascular graft. Therefore, a total of 44/70 (62.8\%) started with a permanent vascular access. On the contrary, $23 / 70(32.8 \%)$ started hemodialysis with a catheter. No association was found between catheter use and age at time of graft loss, gender, and time of graft loss. Patients starting dialysis with a permanent vascular access (fistula or graft) showed a lower mortality compared with patients starting with a catheter. Within the first 30 days 5/23 (21.7 $\%)$ of the catheter group and 3/44 (6.8 \%) patients with a permanent vascular access died. HR of a proportional cox model adjusted for age, early or late graft failure and diabetes showed lower mortality for patients with a permanent vascular access compared to catheter, HR was 0.32 (Cl 95\% 0.12-0.83, p-value 0.019).

\section{Immunosuppression at the time of dialysis initiation and thereafter}

The number of immunosuppressive drugs are illustrated in Figures 6 and 7. In Figure 6 the number of immunosuppressive drugs for patients with and without nephrectomy at the selected point of time after graft loss are shown. The majority of patients (68.6\%) with an allograft in place were on three immunosuppressants at the time of allograft loss (including corticosteroids). Thereafter the number of immunosuppressants was reduced to two, mostly corticosteroids combined with one other immunosuppressant. There was no difference in the early and late graft loss group, regarding the strategy of reduction of 
immunosuppressants. In 4 of the 78 patients, information about immunosuppressive drugs were missing.

After nephrectomy the immunosuppression was reduced to either no immunosuppressants ( $73 \%$ patients) or corticosteroids only (21\% patients). Few nephrectomized patients $(6 \%)$ remained on a second immunosuppressive drug one year after graft loss.

\section{Discussion}

As the protocols for treatment of patients after allograft loss vary widely and little data is available from outside the US, we performed this analysis from the STCS. Over a period of 6.5 years in which 1499 patients received a renal allograft, a total of 78 grafts were lost. This is comparable to the results published in the USRDS (with 7\% in cadaveric donor recipients and $3 \%$ of the living donor recipients, USRDS, 2016). A recent study of 2447 kidney transplantations found that 42 patients died and 67 (2.7\%) lost the graft within a year. The number of early losses 67 of 2447 (2.7\%) was very similar to our study (43 of 1449, 2.8\%) [5].

A striking finding was the high early mortality in our cohort, with 6 out of 41 patients in the early group and 5 out of 37 in the late group. Gill and colleagues described the mortality of patients losing a graft in a cohort transplanted between 1995 and 2003 and compared them with patients on the waiting list, as well as those immediately after transplantation [4]. The death rates were higher in DAGL patients as compared to patients after the major surgery of transplantation (17.9/100 patient-years versus 8.2/100) and was lowest in patients on the waiting list.

An unexpected finding was that the relative mortality was very similar between patients losing the allograft early (i.e. within one year) and late (i.e. after one year). We would have 
expected that the risk of dying after a late allograft loss would be associated with a higher mortality (for various reasons like increased age, time on immunosuppression). This is an important finding as it points towards the danger of the transfer per se and not patient factors. Our data therefore confirm the high early mortality in these patients in a recent cohort and the need for intervention studies to reduce this high mortality.

The vast majority of patients started with hemodialysis after allograft loss. Starting with a native fistula has the lowest morbidity and mortality in incident hemodialysis patients [6]. Unfortunately, there is very little information available about the type of vascular access in DAGL patients. About $55 \%$ of our patients started with a native fistula. The percentage was higher in patients who lost the graft within a year as compared to patients who lost the allograft after one year (59.5\% versus 48,5\%). In one recent study of 61 patients who returned to hemodialysis, $76 \%$ were described to have a native fistula. As an early loss of a graft is an unexpected event, the fistulas were likely still in place from the period before transplantation. Unfortunately, we have not documented whether a fistula was still in place at the time of graft loss in our cohort (and we are not aware of data from the literature). Unexpectedly, the number of native fistulae was even lower in the patients with a late allograft loss and the number of catheters increased significantly. This was particularly the case for the number of patients starting with a tunneled catheter ( $3 \%$ in the early and $18 \%$ in the late group). This indicates a poor pre-dialysis management in the current cohort in patients losing the graft after one year. Consistently, a recent comparison between DAGL patients and incident dialysis patients points towards a poor pre-dialysis care, as DAGL patients had lower bicarbonate, higher phosphate, higher blood pressure, lower albumin and were more likely to be hospitalized after dialysis initiation [7]. 
An important finding was that a permanent vascular access (fistula or graft) was associated with a significantly lower mortality $(\mathrm{HR}=0.32, \mathrm{Cl} 95 \%: 0.12-0.83, \mathrm{p}$-value 0.019$)$. Therefore, an increased use of permanent vascular accesses might be a way to decrease the high mortality. In our experience the loss of allograft function in the late stages of graft failure is difficult to predict and often an unexpected event (e.g. infection) leads to uremic symptoms and necessity of dialysis. Therefore, the timing of discussions about dialysis with an allograft recipient needs to be revisited. Importantly, it should be stated that pre-dialysis information should not be misinterpreted as giving up the allograft. Efforts of prolonging graft function always need to be in parallel with a detailed predialysis work up. Nephrology centres in which the care for renal allograft recipients is separated from the predialysis clinic, need to rethink the management of allograft recipients with poor allograft function.

In our cohort, only three patients started peritoneal dialysis, which is below the countrywide average of incident patients of about $10 \%$. Two detailed analyses from registries from Canada [8] and from the US [9] demonstrated a similar outcome after starting peritoneal dialysis as compared to hemodialysis after graft loss. Therefore, both are viable opportunities and the barriers for PD use need to be addressed. It is well described that a good predialysis information increases the use of PD [10].

How to proceed with immunosuppression after the loss of an allograft is an important question in daily practice. Infections, malignant disease and costs argue for a reduction of immunosuppression [11]. Residual renal function, prevention of immunization and prevention of so-called graft intolerance syndrome are arguments to keep immunosuppression in place.

A survey scrutinized the practice in the US [12]. It illustrated that there were no standards on how to treat DAGL patients, with $75 \%$ of the responders handling immunosuppression 
on an individual decision by the nephrologist in charge. The immunosuppression was rapidly tapered (mostly starting with the calcineurin inhibitor) [12]. 80\% were free of immunosuppression after one year. This is in strong contrast to our swiss cohort. The majority started dialysis with a triple immunosuppression. About $75 \%$ of the patients remained on at least two, the remaining on one immunosuppressive drug. Only after a nephrectomy the immunosuppression was further reduced.

Also there is no good evidence in the literature about the immunosuppression needed to prevent loss of residual renal function, most centers leave some immunosuppression until residual renal function has subsided [13]. In Switzerland the combination of steroids and antimetabolites is most commonly used in this situation. Using prolonged immunosuppression can reduce the risk of immunization by about $40 \%$ [14]. Furthermore, symptoms from graft intolerance syndrome can be avoided, which would result in allograft nephrectomy. In patients not weaned from immunosuppression the rate of hospitalization due to infections increases [15].

An allograft nephrectomy has significant morbidity and mortality, but allows reduction of immunosuppression afterwards without the risk of graft intolerance syndrome [16]. The main indication for an allograft nephrectomy are symptoms of graft intolerance syndrome, particularly not responding to immunosuppression [14]. Allograft nephrectomies are regularly performed in patients losing grafts early. The impact of nephrectomy on outcome like overall mortality and survival of the succeeding graft is difficult to assess due to the interdependence of these factors. Register data suggest an improved survival in patients with allograft nephrectomy but retransplantation is higher in these patients [17]. In one study nephrectomy was associated with a decreased mortality in patients with late allograft loss, but not in patients with early loss [18]. 
A recent meta-analysis which included 1008 patients from 8 studies showed that the nephrectomy group had a longer time interval to retransplantation and a higher rate of positive panel reactive antibody (PRA $>10 \%$ ) before retransplantation [19]. No differences were observed in serum creatinine one year after retransplantation, one-year graft survival rates and one-year patient survival rates. Therefore, no benefit of allograft nephrectomy could be illustrated.

Our cohort describes major differences between the practices in the US and in Switzerland. Prolonged immunosuppression and liberal use of transplant nephrectomies are general practice in Switzerland. The data illustrate an important lack of evidence to guide the treatment in this important phase. Questions have to be addressed in future studies. For patients on the transplant list the benefits and risks of a nephrectomy has to be evaluated. Similarly, in asymptomatic patients who will not be retransplanted slow tapering needs to be tested.

Our data call for randomized controlled trials to improve our knowledge for this important period in the treatment of allograft recipients and an improvement of predialysis care in these patients. 


\section{References}

1. Lacson E, Jr., Wang W, DeVries C, et al. Effects of a nationwide predialysis educational program on modality choice, vascular access, and patient outcomes. American journal of kidney diseases : the official journal of the National Kidney Foundation 2011;58(2):235-242

2. Winkelmayer WC, Mehta J, Chandraker A, et al. Predialysis nephrologist care and access to kidney transplantation in the United States. Am J Transplant 2007;7(4):872-879

3. Aniort J, Kaysi S, Garrouste C, et al. CKD complications in kidney-transplanted patients going back to dialysis: impact on patients outcomes. J Nephrol 2018;31(1):147-155

4. Gill JS, Rose C, Pereira BJ, et al. The importance of transitions between dialysis and transplantation in the care of end-stage renal disease patients. Kidney Int 2007;71(5):442-447

5. van de Luijtgaarden MW, Jager KJ, Segelmark M, et al. Trends in dialysis modality choice and related patient survival in the ERA-EDTA Registry over a 20-year period. Nephrology, dialysis, transplantation : official publication of the European Dialysis and Transplant Association - European Renal Association 2016;31(1):120128

6. Ravani P, Palmer SC, Oliver MJ, et al. Associations between Hemodialysis Access Type and Clinical Outcomes: A Systematic Review. J Am Soc Nephrol 2013;24(3):465-473

7. Liakopoulos V, Roumeliotis S, Gorny X, et al. Oxidative Stress in Patients Undergoing Peritoneal Dialysis: A Current Review of the Literature. Oxidative medicine and cellular longevity 2017;2017:3494867

8. Perl J, Hasan O, Bargman JM, et al. Impact of dialysis modality on survival after kidney transplant failure. Clin J Am Soc Nephrol 2011;6(3):582-590

9. Perl J, Dong J, Rose C, et al. Is dialysis modality a factor in the survival of patients initiating dialysis after kidney transplant failure? Perit Dial Int 2013;33(6):618-628

10. Manns BJ, Taub K, Vanderstraeten C, et al. The impact of education on chronic kidney disease patients' plans to initiate dialysis with self-care dialysis: a randomized trial. Kidney Int 2005;68(4):1777-1783

11. Sommerer $\mathrm{C}$, Konstandin $\mathrm{M}$, Dengler $\mathrm{T}$, et al. Pharmacodynamic monitoring of cyclosporine a in renal allograft recipients shows a quantitative relationship between immunosuppression and the occurrence of recurrent infections and malignancies. Transplantation 2006;82(10):1280-1285

12. Bayliss GP, Gohh RY, Morrissey PE, et al. Immunosuppression after renal allograft failure: a survey of US practices. Clin Transplant 2013;27(6):895-900

13. Elmahi N, Csongradi E, Kokko K, et al. Residual renal function in peritoneal dialysis with failed allograft and minimum immunosuppression. World J Transplant 2013;3(2):26-29

14. Augustine JJ, Woodside KJ, Padiyar A, et al. Independent of nephrectomy, weaning immunosuppression leads to late sensitization after kidney transplant failure. Transplantation 2012;94(7):738-743

15. Woodside KJ, Schirm ZW, Noon KA, et al. Fever, infection, and rejection after kidney transplant failure. Transplantation 2014;97(6):648-653

16. Akoh JA. Transplant nephrectomy. World J Transplant 2011;1(1):4-12

17. Ayus JC, Achinger SG, Lee $S$, et al. Transplant nephrectomy improves survival following a failed renal allograft. J Am Soc Nephrol 2010;21(2):374-380

18. Johnston $\mathrm{O}$, Rose $\mathrm{C}$, Landsberg $\mathrm{D}$, et al. Nephrectomy after transplant failure: current practice and outcomes. Am J Transplant 2007;7(8):1961-1967

19. Wang K, Xu X, Fan M, et al. Allograft nephrectomy vs. no-allograft nephrectomy for renal transplantation: a meta-analysis. Clin Transplant 2016;30(1):33-43

Acknowledgements: This work was supported by a grant from 
Tables:

Table 1: Baseline characteristics of the study cohort

\begin{tabular}{|c|c|c|}
\hline & $\begin{array}{l}\text { Patients without allograft } \\
\text { loss }\end{array}$ & Patients with allograft loss \\
\hline Number of patients & 1421 & 78 \\
\hline Number of males (\%) & $906(63.8)$ & $57(73.1)$ \\
\hline $\begin{array}{l}\text { Age at Transplantation (years, } \\
\text { median (IQR)) }\end{array}$ & $54(44.6)$ & $57(44.7)$ \\
\hline $\begin{array}{l}\text { Observation time days since } \\
\text { Transplantation (median, IQR) }\end{array}$ & $1445(729,1907)$ & $1191(447,2257)$ \\
\hline First Allograft (\%) & $1197(84.2)$ & $56(71.8)$ \\
\hline Re-Transplantation (\%) & $224(15.8)$ & $22(28.2)$ \\
\hline \multicolumn{3}{|l|}{ Donor } \\
\hline Brain dead (\%) & $736(51.8)$ & $60(77)$ \\
\hline Living (\%) & $637(44.8)$ & $16(20.5)$ \\
\hline Non-heart-beating donor (\%) & $48(3.4)$ & $2(2.5)$ \\
\hline \multicolumn{3}{|l|}{ Dialysis before transplantation } \\
\hline HD (\%) & $969(68.2)$ & 64 (82.1) \\
\hline PD (\%) & $199(14)$ & $9(11.5)$ \\
\hline Pre-emptive (\%) & $252(17.7)$ & $5(6.4)$ \\
\hline Unknown (\%) & $1(0.1)$ & 0 \\
\hline \multicolumn{3}{|l|}{ Etiology (number, \%) } \\
\hline Glomerulonephritis / Vasculitis & $433(30.5)$ & 26 (33.3) \\
\hline Polycystic kidney disease & $282(19.9)$ & $12(15.4)$ \\
\hline $\begin{array}{l}\text { Hypertensive / renovascular } \\
\text { nephrosclerosis }\end{array}$ & $183(12.9)$ & $11(14.1)$ \\
\hline Diabetic nephropathy & $116(8.2)$ & $5(6.4)$ \\
\hline $\begin{array}{l}\text { Obstructive nephropathy / Reflux } \\
\text { / Pyelonephritis }\end{array}$ & $85(6)$ & $4(5,1)$ \\
\hline $\begin{array}{l}\text { Hereditary kidney disease other } \\
\text { than polycystic kidney disease }\end{array}$ & $45(3.2)$ & $2(2.6)$ \\
\hline Interstitial nephritis, not hereditary & $46(3.2)$ & $2(2.6)$ \\
\hline $\begin{array}{l}\text { Congenital disease / } \\
\text { malformation }\end{array}$ & $40(2.8)$ & $3(3.9)$ \\
\hline Other & $344(24.2)$ & $24(30.8)$ \\
\hline \multicolumn{3}{|l|}{ Comorbidities } \\
\hline Skin cancer & $85(6)$ & $3(3.9)$ \\
\hline Cancer other than skin & $152(10.7)$ & $13(16.7)$ \\
\hline Cardiopulmonary diseases & 709 (49.9) & $47(60.3)$ \\
\hline Infectious diseases & $303(21.3)$ & $17(21.8)$ \\
\hline Diabetes mellitus & $197(13.9)$ & $11(15.4)$ \\
\hline
\end{tabular}


Table 2: Information about patients who lost the allograft

\begin{tabular}{|l|l|l|l|}
\hline & $\begin{array}{l}\text { All with } \\
\text { allograft loss }\end{array}$ & $\begin{array}{l}\text { Allograft loss } \\
\text { within one year }\end{array}$ & $\begin{array}{l}\text { Allograft loss } \\
\text { after one year }\end{array}$ \\
\hline Number of patients & 78 & $41(53)$ & $37(47)$ \\
\hline Number of males (\%) & $57(73)$ & $30(73)$ & $27(73)$ \\
\hline $\begin{array}{l}\text { Age at transplantation in years } \\
\text { (median (IQR)) }\end{array}$ & $57(46,66)$ & $60(51,67)$ & $50(34,64)$ \\
\hline $\begin{array}{l}\text { Observation time in days since } \\
\text { graft loss (median, IQR) }\end{array}$ & $711(323,1364)$ & $446(137,925)$ & $\begin{array}{l}790(441, \\
1468)\end{array}$ \\
\hline $\begin{array}{l}\text { Time between transplantation } \\
\text { and graft loss in days (median, } \\
\text { IQR) }\end{array}$ & $300(21,864)$ & $28(3,130)$ & $\begin{array}{l}923(501, \\
1404)\end{array}$ \\
\hline $\begin{array}{l}\text { Number of patients with allograft } \\
\text { loss within 30 days (\%) }\end{array}$ & $21(27)$ & $21(27)$ & \\
\hline Relisting n (\%) & & $6(14.6)$ & $2(5.4)$ \\
\hline No & $8(10.3)$ & $9(22.0)$ & $17(46.0)$ \\
\hline Yes & $26(33.3)$ & $26(63.4)$ & $18(48.7)$ \\
\hline Unknown & $44(56.4)$ & & \\
\hline
\end{tabular}


Table 3: Causes of allograft nephrectomy $(n=50)$

\begin{tabular}{|l|c|c|}
\hline & $\mathrm{n}$ & $\%$ \\
\hline Infections & 12 & 24 \\
\hline Rejection & 10 & 20 \\
\hline BK nephropathy & 4 & 8 \\
\hline Cancer & 1 & 2 \\
\hline Other & 18 & 36 \\
\hline
\end{tabular}

Multiple reasons possible 
Table 4: Dialysis forms and access after allograft loss

\begin{tabular}{|c|c|c|c|}
\hline & $\begin{array}{c}\text { All with allograft } \\
\text { loss (n = 78) }\end{array}$ & $\begin{array}{c}\text { Allograft loss within } \\
\text { one year (n = 41) }\end{array}$ & $\begin{array}{l}\text { Allograft loss after one } \\
\text { year (n = 37) }\end{array}$ \\
\hline Hemodialysis & 70 & $37(90.2)$ & $33(89.2)$ \\
\hline Native fistula & $38(54.3)$ & $22(59.5)$ & $16(48.5)$ \\
\hline $\begin{array}{l}\text { Arteriovenous } \\
\text { Graft }\end{array}$ & $6(8.6)$ & $4(10.8)$ & $6(18.2)$ \\
\hline $\begin{array}{l}\text { Tunneled } \\
\text { catheter }\end{array}$ & $7(10)$ & $1(2.7)$ & $7(21.2)$ \\
\hline $\begin{array}{c}\text { Non-tunneled } \\
\text { catheter }\end{array}$ & $16(22.9)$ & $9(24.3)$ & $2(5.4)$ \\
\hline $\begin{array}{l}\text { Missing } \\
\text { information on } \\
\text { access }\end{array}$ & $3(4.2)$ & $1(2.4)$ & $1(2.7)$ \\
\hline Peritoneal dialysis & 3 & $2(4.9)$ & $1(2.7)$ \\
\hline $\begin{array}{l}\text { No renal replacement } \\
\text { therapy }\end{array}$ & 2 & $1(2.4)$ & $2(5.4)$ \\
\hline $\begin{array}{l}\text { Missing information } \\
\text { on treatment }\end{array}$ & 3 & $1(2.4)$ & \\
\hline
\end{tabular}


Legends

Figures

Figure 1: Study population. 1499 patients received a renal allograft during the observation time. 82 allografts were lost during the follow-up period. 4 patients were lost to follow-up. Therefore, detailed information was given on 78 patients.

Figure 2: Patient courses after allograft loss (A within one year, B after one year). Each patient is illustrated as a horizontal line with the events deaths (D), nephrectomy $(N)$, relisting (L) and retransplantation $(\mathrm{R})$ illustrated in the course.

Figure 3: Percentage of patients with events after allograft loss ( $A$ in all patients, $B$ with allograft loss within one year, $\mathrm{C}$ with allograft loss after one year). Note the same early death rate in both groups. All but one patient were transplant nephrectomized within a year in the early loss group (B), whereas this reached $80 \%$ after 2 years in late loss group.

\section{Figure 4: Survival probability after allograft loss}

Figure 5: Cumulative incidence of allograft nephrectomies. Note that in almost all patients who lost the allograft within a year the allograft was removed within half a year after graft loss. Overall, about $60 \%$ received an allograft nephrectomy.

Figure 6: Immunosuppression after graft loss. Illustrated are the percentages of patients (with and without nephrectomy) according to the number of immunosuppressive drugs. In the cohort, the number of immunosuppressive drugs does not change significantly as long as the 
allograft is in place. Even at the time of allograft loss the majority is still under triple immunosuppressive therapy (nr: number, mth: months).

Figure 7: Immunosuppressive drugs and glucocorticoid therapy. Illustrated are the number of patients according to the number of immunosuppressant. Glucocorticoid use is illustrated. Note that the vast majority was on glucocorticoids. Only after nephrectomy the overall number of immunosuppressants decreases. 